\title{
News and Events
}

\author{
Museum of Finnish Architecture - Exhibitions \\ Art for the State (runs until 16 February 1997) \\ Contemporary Finnish Architects (runs until 16 \\ February 1997) \\ Hilding Ekelund (Spring 1997)
}

Information: The Museum of Finnish Architecture, Kasarmikatu 24, 00130 Helsinki, Finland.

Tel: 661918. Fax: 662573.

Second International Symposium on Urban Planning and Environment: Strategies and Methods for Improving Environmental Quality in Compact Cities

11-14 March 1997, Groningen, The Netherlands

Information: UPE Symposium Organizing Committee, Faculty of Spatial Sciences, University of Groningen, P.O. Box 800, 9700 AV Groningen, The Netherlands.

Fax: 00315036 33901. Email: upe@frw.rug.nl

Web site: http://www.frw.rug.nt/upe.html

First International Space Syntax Symposium

16-18 April 1997, University College, London

Information: Symposium Organizer, Mark David Major, The Bartlett School of Graduate Studies, 1-19 Torrington Place, University College London, Gover Street, London WC1E 6BT

28th Annual Conference of the Environmental Design Research Association: Space Design and Management for Place-making

7-11 May 1997, Université du Québec, Montréal, Canada

Information: Maurice Amiel, Université du Québec à Montréal, Département de design, Case postale 8888, Succursale Centre-ville, Montréal, Québec H3C 3P8, Canada

Tel: (514) 9873000 ext. 3911. Fax: (514) 9877717. Email: Vischer@accent.net.

\section{Fourth International Seminar on Urban Form}

18-21 July 1997, University of Birmingham, UK

Information: Professor J. W. R. Whitehand, Urban Morphology Research Group, School of Geography, University of Birmingham B15 2TT.

Tel: 0121414 5536. Fax: 01214145528.

Email: umrg@bham.ac.uk

Oxford Women's Studies Network Conference: Women and the City

20 September 1997, Oxford Brookes University, Oxford, UK

Information: Sue Ledwith, School of Business, Oxford Brookes University, Wheatley, Oxford OX33 1HX

\section{Cities in Crisis}

3 October 1997, London Docklands, UK

Information: Gerald Cary-Elwes, Secretary General, BURA or Andrew Carter, Policy Researcher, BURA.

Tel: 0171253 5054. Fax: 01714908735.

European Architectural Competition: on the theme - New collective spaces in the contemporary city

Submission of entries by 24 March 1997

Information: Competition secretariat: Europan/ Thessaloniki 1997, The Organization for the Cultural Capital of Europe-Thessaloniki 1997, 105 Vassilissis Olgas Avenue, 54643 Thessaloniki, Greece. Tel: 093 268143. Fax: 3031867870. 